\title{
Nuevas narrativas aplicadas al periodismo colaborativo de investigación
}

\author{
Dr. David Parra Valcarce|davidparra@ccinf.ucm.es \\ Universidad Complutense de Madrid \\ Dra. Concha Edo Bolós|conchaed@ccinf.ucm.es \\ Universidad Complutense de Madrid \\ Dr. Juan Carlos Marcos Recio|jmarcos@ucm.es \\ Universidad Complutense de Madrid
}

\author{
Palabras clave \\ Periodismo colaborativo; nuevas narrativas, ICIJ; \\ ProPublica; Journalism++. \\ Sumario \\ 1. Introducción. 2. Metodología. 3. Resultados. 4. \\ Discusión y conclusiones. 5. Bibliografía. 6 . \\ Financiación
}

\begin{abstract}
Resumen
La difusión internacional de los "Papeles de Panamá" desde abril de 2016 abre un nuevo ámbito de actuación en el periodismo colaborativo de investigación: el proceso de cooperación que se establece entre distintas organizaciones dedicadas a la
\end{abstract} generación de contenidos informativos, cuyo objetivo estratégico es superar las carencias detectadas en los medios de comunicación en ámbitos como el financiero, el de la calidad de contenidos o el de su impacto social, entre otros. Este artículo examina las bases estratégicas que permiten la puesta en marcha de distintos proyectos y analiza las nuevas narrativas aplicadas a tres de las experiencias que, hasta el momento, han tenido un mayor éxito: International Consortium of Investigative Journalists (ICIJ), ProPublica y Journalism++. Como principales conclusiones, estas propuestas demuestran la creciente solidez del periodismo colaborativo de investigación, la diversidad de tipologías que admite dicho formato y la constante apuesta por nuevas narrativas de carácter multimedia y por nuevos procedimientos para presentar las ingentes cantidades de datos que son necesarias para generar contenidos periodísticos de calidad.

\section{Cómo citar este texto:}

David Parra Valcarce, Concha Edo Bolós y Juan Carlos Marcos Recio (2017): "Nuevas narrativas aplicadas al periodismo colaborativo de investigación", en Miguel Hernández Communication Journal, nº, pp. 591 a 616. Universidad Miguel Hernández, UMH (ElcheAlicante). Recuperado el $\_$de de 20

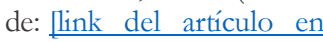
mhjournal.org] 


\title{
New narrative skills applied to research collaborative journalism
}

\author{
Dr. David Parra Valcarce|davidparra@ccinf.ucm.es \\ Universidad Complutense de Madrid \\ Dra. Concha Edo Bolós|conchaed@ccinf.ucm.es \\ Universidad Complutense de Madrid \\ Dr. Juan Carlos Marcos Recio|jmarcos@ucm.es \\ Universidad Complutense de Madrid
}

\section{Keywords}

Collaborative Journalism; new narrative skills;

ICIJ; ProPublica; Journalism++.

\section{Summary}

1. Introduction. 2. Methodology. 3. Results. 4.

Discusssion and conclusions. 5. Bibliography. 6.

Financing

\section{Abstract}

The international spreading of "Panama Papers" since April 2016 opens a new field of action in research collaborative journalism: the cooperation process that is established between several organizations dedicated to the generation of information contents, whose strategic objective is overcoming the shortcomings detected in mass media in areas such as financial, content quality or social impact, among others. This article examines the strategic bases that allow the implementation of different projects and

analyzes the new narratives skills applied to the three ones experiences that, to date, have been most successful: International Consortium of Investigative Journalists (ICIJ), ProPublica and Journalism ++. As main conclusions, these proposals demonstrate the growing strength of collaborative investigative journalism, the diversity of typologies supported by this format and the constant commitment to new multimedia narratives and new procedures to present the huge amounts of data needed to generate qualified journalistic contents.

How to cite this text:

David Parra Valcarce, Concha Edo Bolós y Juan Carlos Marcos Recio (2017): “New narrative skills applied to research collaborative journalism", en Miguel Hernández Communication Journal, no8, pp. 591 to 616, Universidad Miguel Hernández, UMH (Elche-Alicante). Accessed 20 _ in: [paper link in mhjournal.org] 


\section{Introducción}

El comienzo de la segunda década del siglo XXI marca un punto de inflexión en el devenir del ecosistema de la información. Coinciden en el tiempo diferentes circunstancias de particular relevancia para explicar este profundo cambio. Entre ellas destacamos: la llegada de una nueva generación de consumidores de contenidos periodísticos cuyo patrón de aprendizaje es marcadamente audiovisual y multimedia, por oposición al procedimiento clásico que se sustentaba en la lectura del texto impreso; la gradual conversión del negocio de la información en un mercado de oferta frente al tradicional mercado de demanda de décadas atrás; o la consolidación del peso específico de las nuevas tecnologías de la información y las comunicaciones (NTIC) en los procesos de producción, almacenamiento y distribución de toda clase de contenidos.

También conviene tener en cuenta: el desplazamiento del consumidor de información en beneficio de la figura del prosumidor (Toffler, 1980 y 1990; Bruns, 2006 y 2009; Bird, 2011) de contenidos, cada vez más crítico con la actividad desarrollada por los medios de comunicación y con los profesionales de la información que trabajan en ellos; la puesta en entredicho del modelo de negocio paradigmático del periodismo, cuya estructura de ingresos estaba fuertemente influida por los procedentes de la comercialización de espacio/tiempo puestos a disposición de los anunciantes; una honda crisis económica a escala internacional, que afecta a la totalidad de los sectores productivos y cuyos verdaderos efectos habrán de ser analizados en el medio plazo; y un crecimiento del consumo de información en redes sociales que ha facilitado la difusión y la aceptación de la posverdad.

Esta transformación tecnológica hacia la digitalización afecta a modelos de negocio y gestión de las empresas periodísticas e incide sobre la manera en que los medios de comunicación ofrecen los contenidos a sus audiencias (Gómez Mompart, Gutiérrez Lozano y Palau, 2015: 144). 
En su análisis World Press Trends 2015 correspondiente a junio de 2015 la World Association of Newspapers and News Publishers (WAN-IFRA) pone de relieve algunas de las estrategias adoptadas por los medios de comunicación para afrontar este conjunto de cambios y desafíos. Dichas estrategias son de la más variada índole e incluyen desde el completo abandono del negocio hasta la apuesta en exclusiva por el formato online, pasando por la compatibilización entre los soportes papel y digital y/o la adecuación a las nuevas generaciones de soportes móviles (compuesta principalmente por teléfonos móviles inteligentes, tabletas electrónicas y dispositivos híbridos) bien con aplicaciones dedicadas bien mediante diseños responsivos.

De manera paralela, nos encontramos ante lo que otros autores, seguidos con posterioridad por una extensa lista de investigadores sobre la materia, califican como el entorno Web 2.0. En un caso, advertimos una recalificación de la noción más tradicional de la Red (DiNucci, 1999: 32) mientras que en otras ocasiones se incide sobre la noción de web semántica manual (O’Reilly, 2005) o sobre aspectos que tienen que ver con todo lo que atañe al fenómeno de la socialización de la información (García Galera y Del Hoyo, 2013: 114).

Tradicionalmente las bases conceptuales del periodismo de investigación se sitúan en revelar cuestiones encubiertas bien de manera deliberada, generalmente por alguien que se encuentra en una posición de poder, bien de forma accidental ante la existencia de una masa caótica de datos y circunstancias, así como en el posterior análisis y exposición pública de todos los datos relevantes.

Parasie (2015), a partir de las investigaciones de Ettema y Glasser (1998), establece tres de los principales elementos de su epistemología (Parasie, 2015: 367): enfoque externo de la verdad, pluralidad de fuentes de información y surgimiento simultáneo de hechos y relato durante el transcurso de la investigación. 
Desde el punto de vista formal el periodismo de investigación se caracteriza por ir más allá de la estructura típica de una noticia sustentada en las cinco W (quién, qué, cuándo, dónde y por qué) dado que además de incluir tales elementos apuesta por "involucrar personajes que tienen motivaciones, características físicas, historias personales y otras características que exceden cualquier título u opinión. La investigación se lleva a cabo en lugares con personajes específicos e historias propias, y nos muestra un pasado donde comenzó la historia, un presente donde ésta se ha revelado y un futuro que será resultado de la revelación" (Hunter, 2013: 66).

Hasta comienzos del siglo XXI el periodismo de investigación fue liderado por las grandes empresas periodísticas, las únicas con capacidad financiera para asumir sus ingentes costes económicos, y volcado en el soporte del periodismo impreso. Pero el cambiante ecosistema de la información al que anteriormente se aludía propicia la aparición de novedosas iniciativas y proyectos de actuación (Bradshaw, 2008: 109).

Nos parece de particular relevancia la alusión realizada por Shirky a tres niveles de colaboración en los procesos de producción y distribución de los contenidos informativos, dentro de un reparto de competencias que resulta plenamente aplicable al ámbito del periodismo de investigación. En el primero de los niveles se produce un mero proceso de compartir contenidos; el segundo lleva implícita la producción colaborativa, que implica tanto que haya distintos grupos de trabajo preestablecidos como que dichos grupos operen bajo procedimientos y propósitos comunes; y el último alude a la acción colectiva, que conlleva acciones estratégicas conjuntas, integradas y al unísono. Es en este tercer nivel donde cabe referirse a una transformación verdaderamente significativa, tal y como expone el propio autor (Shirky, 2008: 160): "Revolution doesn't happen when society adopts new technologies -it happens when society adopt new behaviours". 
En un entorno de esta índole surge un fenómeno como es el del periodismo de investigación colaborativo, entendido como el proceso de cooperación que se establece entre distintas organizaciones dedicadas a la generación de contenidos informativos de investigación, cuyo objetivo estratégico es superar las actuales carencias detectadas en los medios de comunicación en ámbitos como el financiero, el de la calidad de contenidos o el de la recuperación de la credibilidad social, entre otros. Dicha fórmula permite afrontar una realidad caracterizada por una ampliación de la "geografía del consumo en un escenario de quioscos y territorios globales" (Díaz Nosty, 2013: 36).

\section{Metodología}

\subsection{Consideraciones generales}

Para proceder al análisis de las fórmulas y modelos de aplicación de las nuevas narrativas al periodismo colaborativo de investigación se ha procedido, en primer lugar, a una revisión bibliográfica que permita una mejor comprensión del contexto que fomenta el surgimiento de dicho fenómeno, la delimitación de las etapas que marcan su devenir a lo largo de esta última década y la determinación de los niveles de cooperación que abarca.

El examen de la literatura científica posibilita entender de qué manera el uso de esas nuevas narrativas contribuye a sustentar el periodismo colaborativo de investigación y superar determinadas carencias estratégicas financieras, de impacto social sobre la opinión pública o de calidad y relevancia de contenidos.

Para la elección de los ejemplos de periodismo colaborativo de investigación se han tenido en cuenta los trabajos de autores como Gershenfeld, Krikorian y Cohen, quienes a su vez beben de las investigaciones planteadas por Baran y Licklider en la década de los sesenta del siglo XX, dado que permiten una mejor comprensión de la evolución de Internet desde una mera infraestructura de comunicaciones a un complejo entorno que propicia compartir toda clase de contenidos de manera transnacional. 


\subsection{Definición del método}

Nuestro punto de partida para el análisis de los proyectos de referencia es el año 1999, fecha de publicación de The Cluetrain Manifesto, un documento compuesto por 95 tesis que intenta llamar la atención sobre la incidencia de Internet sobre corporaciones y ciudadanos. El texto pone particular énfasis en que es necesario llevar a cabo diferentes transformaciones de muy hondo calado para afrontar los retos de un mercado muy dinámico y cambiante. La trigésima primera de las tesis sintetiza este espíritu: "Networked markets can change suppliers overnight. Networked knowledge workers can change employers over lunch. Your own 'downsizing initiatives' taught us to ask the question: 'Loyalty? What's that?”’.

En gran medida este singular entorno se halla muy influido por una nueva generación de usuarios, entre cuyas pautas diferenciales de consumo se distinguen la concepción lúdica de la existencia, una menor cantidad de tiempo libre, la fragmentación social del consumo, las nuevas fórmulas laborales y familiares, un proceso de consumo basado en el crédito, la búsqueda permanente de la oferta, su carácter transnacional y la supresión de los tradicionales límites temporales (Sabés y Parra, 2014: 513).

El examen de las propuestas concretas de nuevas narrativas llevadas a cabo en los proyectos seleccionados tiene bien presente que no debe entenderse como algo limitado a una determinada estética formal, a una propuesta multimedia específica o a un estilo de relato narrativo concreto. Por supuesto implica las realidades anteriormente indicadas pero también atañe a aspectos que tienen que ver con los procesos de obtención, gestión, tratamiento y presentación formal de las ingentes cantidades de datos que son necesarias para generar contenidos periodísticos de calidad en forma de reportajes de investigación en profundidad.

En uno u otro caso, lo que sí constituye un eje directriz común es la activa aplicación de las nuevas tecnologías de la información y las comunicaciones, 
incluyendo el empleo tanto de herramientas de software de carácter propietario como de otras sustentadas en código de acceso libre, puestas al servicio de ese novedoso modelo de generación de contenidos (Moloney, 2011).

Por consiguiente, se ha optado por un análisis cualitativo de algunos proyectos de narrativa transmedia particularmente relevantes en todos y cada uno de los casos elegidos, examinando sus singularidades formales y de contenido, los elementos organizativos que implica su puesta en marcha y desarrollo, la variada estructura de colaboración entre diferentes empresas de la información que conlleva cada iniciativa y los condicionamientos para su publicación final por parte de los medios de comunicación implicados en este conjunto de procedimientos.

\subsection{Selección de la muestra}

Se ha prestado particular atención a tres de estos proyectos de carácter transnacional, por considerar que representan la esencia del proceso que estamos aquí examinando. Los tres proyectos considerados son los referidos a International Consortium of Investigative Journalists (ICIJ), ProPublica y Journalism ++ . El primero alude a una entidad surgida con anterioridad a Internet, aunque cobra toda su relevancia en el entorno Web 2.0, mientras que las dos restantes sí se centran ya en organizaciones nativas digitales.

Puede advertirse que en los tres proyectos se percibe la forma en que el periodismo de investigación resulta particularmente proclive al establecimiento de iniciativas colaborativas, esencialmente por la concatenación de tres circunstancias estratégicas: generar un contenido que sería inviable sin la participación de un determinado número de autores; compartir los costes económicos optimizando el nivel de inversiones requeridas; y conseguir los máximos niveles posibles de difusión entre la opinión pública, implicando a numerosos medios, al menos representativo por cada país. 
La consecución de estos objetivos pasa, entre otros aspectos, por la apuesta por relatos diferentes de los habituales en esta clase de contenidos, en buena medida enraizados con la noción de narrativa transmedia, conforme a lo expuesto por autores como Jenkins (2009) o Scolari (2013) y que identifican dicha narrativa con "la búsqueda de la participación del lector/espectador quien, al menos, formará parte de la estrategia de difusión de la pieza a través de sus recomendaciones, comentarios o redifusiones" (Irala, 2014: 147).

\subsubsection{International Consortium of Investigative J ournalists}

El International Consortium of Investigative Journalists (ICIJ) es una entidad que forma parte del Center for Public Integrity, una organización surgida en Estados Unidos en 1977. Como tal consorcio, el ICIJ se creó en 1997 con el propósito de dotar de recursos a periodistas de investigación de todo el mundo. Dichos recursos son de una triple naturaleza: tecnología de última generación, financiación económica y cobertura jurídica.

Con sede en Washington, en la actualidad cuenta con cerca de 200 periodistas pertenecientes a 65 países, todos ellos inmersos en distintos proyectos colaborativos de investigación de la más variada índole. Desde el punto de vista estratégico apuesta por un empleo de la colaboración entre los profesionales de la información que permita su utilización para la construcción de diálogos transacciones en los reportajes de investigación.

El ICIJ fue la primera organización en plantear la posibilidad de que entidades de distintos países trabajasen en conjunto en un escenario cada vez más globalizado, anticipándose así a cuestiones como el creciente coste económico de este tipo de contenidos o la puesta en tela de juicio del tradicional modelo de negocio del mercado de la información.

La financiación del consorcio, cuyo presupuesto anual ronda el millón de dólares, se basa en donantes individuales (con ap ortaciones mínimas de 10 
dólares y máximas de 1.000 dólares salvo casos excepcionales) y en los fondos proporcionados por determinadas entidades filantrópicas como Adessium Foundation, Open Society Foundations, The Sigrid Rausing Trust, The Ford Foundation, The David and Lucile Packard Foundation, Pew Charitable Trusts o Waterloo Foundation. Todas las investigaciones que son generadas se comparten de manera gratuita en Internet y pueden ser difundidas sin limitaciones por cualquier medio de comunicación.

La difusión de los conocidos como Papeles de Panamá a partir de abril de 2016 marca un antes y un después en el devenir del ICIJ. La relevancia adquirida, junto al considerable aumento en el número de donantes a su actividad cotidiana, han servido para garantizar su existencia como una entidad plenamente independiente en el corto y medio plazo.

No obstante, en octubre de 2016 anuncia la separación del Centro de Integridad Pública (CPI) con el fin de que "ambos equipos periodísticos aprovechen el impacto masivo que han tenido como organización y permitir que cada uno busque nuevas oportunidades y opciones para financiar y llevar a cabo su crucial trabajo" (Berry, 2016). Precisamente cuando tenían más actividades en conjunto ha llegado esta ruptura, en la que han influido aspectos de naturaleza especialmente financiera. El proceso se ha consumado en febrero de 2017.

El director durante los últimos cinco años apunta que desde el 24 de febrero de 2017 el ICIJ es una organización de noticias sin fines de lucro totalmente independiente, con sede en Washington y comprometida con la producción de los mejores informes de investigaciones transfronterizos en el mundo. Y manifiesta su agradecimiento al Centro de Integridad Pública por sus diecinueve años de colaboración y su apoyo continuo (Ryle, 2017). 


\subsubsection{ProPublica}

ProPublica es una entidad creada a finales de 2007 en Estados Unidos gracias al impulso de Paul Steiger, antiguo editor de The Wall Street Journal. Se estructura como una institución sin ánimo de lucro que ha apostado por un modelo que combina la filantropía con la colaboración estratégica (no tanto de generación de contenidos como económica) con otras empresas de la información. Sus ingresos proceden de tres vías: fondos aportados por la Sandler Foundation, donaciones privadas y publicidad. Dichos ingresos, conforme a los datos ofrecidos en su informe anual correspondiente al ejercicio 2016, se sitúan por encima de los 17,2 millones de dólares (ProPublica, 2017).

Liderada por Stephen Engelberg y Richard Tofel, periodistas procedentes de The Oregonian y The Wall Street Journal respectivamente, en la actualidad tiene 45 periodistas en plantilla y ha establecido acuerdos de colaboración con 139 socios estratégicos. Su número de donantes supera los 26.000 en todo el mundo.

Su modo de funcionamiento se basa en tres grandes ejes directrices: apostar por una plantilla fija (se aleja del modelo freelance) que se dedica a la investigación de los temas sin tener la presión económica que podría tener un profesional de tales características; contar con dos tipos de socios, los publicadores (con derecho a publicar en condiciones de exclusividad determinados reportajes previamente determinados) y los difusores (reproducen los contenidos siempre bajo licencia Creative Commons y sin exclusividad); y adoptar la opción estratégica de que los pormenores de cualquier investigación quedan estrictamente restringidos al equipo de periodistas de la redacción propia, no permitiendo el acceso de colaboradores, donantes o anunciantes a los contenidos mientras son investigados.

A lo largo de sus años de existencia ProPublica se ha caracterizado por desarrollar un conjunto de contenidos basados en el periodismo de 
investigación que tratan de sacar a la luz y difundir entre la sociedad los abusos del poder y las amenazas al bien público procedentes de gobiernos, grandes corporaciones y otras instituciones.

Gracias a ello en 2010 se convirtió en la primera organización nativa digital en obtener un Premio Pulitzer, gracias al reportaje titulado The Dealy Choices at Memorial, publicado en agosto de 2009 en The New York Times. En dicho reportaje, narrado en primera persona por la periodista y médico Sheri Fink, se analizaban las decisiones de un grupo de médicos que atendían a pacientes terminales en los días posteriores a que el huracán Katrina devastara la ciudad de New Orleans. Al año siguiente fue también la primera en conseguir un nuevo Premio Pulitzer por un reportaje publicado en soporte exclusivamente online gracias a un conjunto de relatos bajo el título genérico de The Wall Street Money Machine, donde se examinaban (y denunciaban) determinadas prácticas escasamente deontológicas de entidades financieras estadounidenses.

\subsubsection{J ournalism ++}

Journalism ++ , de origen europeo, tiene como impulsores al desarrollador informático Pierre Romera y al periodista Nicolas Kayser-Bril, procedentes del portal Owni especializado en la elaboración de reportajes en profundidad. En un primer momento sus sedes se encontraban en Berlín y París y con posterioridad su radio de acción se ha extendido, en forma de franquicia, a las ciudades de Ámsterdam, Colonia, Estocolmo y Oporto, a las que se añade la de Sao Paulo.

Surgida en 2011, se define como una empresa dedicada a la elaboración de contenidos periodísticos que son generados por unos recursos humanos que suponen una mezcla entre desarrolladores de tecnologías de la información, diseñadores y periodistas. El formato paradigmático es el de proyectos de historias sustentadas en datos. En su manifiesto fundacional se subraya (Journalism++, 2017): "We define journalism as making interesting what is important and we will refrain from making important what is interesting". 
A diferencia de ICIJ o ProPublica, Journalism++ sí contempla el ánimo de lucro en sus procedimientos operativos. Sus ingresos provienen de una combinación entre servicios proporcionados a organizaciones de medios y premios para la realización de proyectos. No obstante, a fecha de hoy dichos ingresos no han sido hecho públicos.

Otro elemento de diferenciación es la mezcla entre la faceta propiamente periodística y la que se sustenta en las nuevas tecnologías de la información y las comunicaciones aplicadas a los procesos de obtención, comprensión y formalización de contenidos sustentados en gigantescas cantidades de datos. Tal y como se comprobará a continuación, esa vertiente que linda con una realidad como los Big Data (y que va más allá de la mera noción de periodismo de datos o periodismo de precisión) marca unos procedimientos narrativos característicos que tienen mucho que ver con los siete rasgos del relato transmedia expuestos por Jenkins (2009): expansión vs. profundidad; continuidad vs. multiplicidad; inmersión vs. extracción; construcción de mundos; serialidad; subjetividad; y ejecución.

\section{Resultados}

\subsection{International Consortium of Investigative J ournalists}

Como se indicó con anterioridad, uno de los principales valores añadidos de formar parte del International Consortium of Investigative Journalists es el acceso a tecnología de última generación incorporada al servicio del periodismo de investigación.

Desde el punto de vista de la utilización de nuevos formatos narrativos el ICIJ atrajo la atención de la opinión pública internacional ya en la segunda década del siglo XXI con motivo de dos series de reportajes colaborativos: en primer lugar los referidos a la temática de SwissLeaks, un proyecto que implicó a más de cincuenta medios de comunicación y que permitió dar a conocer los nombres de miles de clientes de la filial suiza del banco HBSC que habían 
evadido impuestos durante las últimas décadas; y, en segundo término, el asunto Skin and Bone, sobre el tráfico de tejidos humanos con víctimas procedentes de países en vías de desarrollo.

Es verdad que en ambos casos los asociados, como en cualquier proyecto liderado desde esta organización, dispusieron de plena libertad formal para la adaptación de sus respectivos relatos periodísticos. Pero no es menos cierto que podemos encontrar algunos elementos comunes en cuanto a esta novedosa propuesta narrativa se refiere.

En lo que atañe a Swiss Leaks se trataba de una estructura esencialmente basada en el texto escrito, con escasa presencia de gráficos o infografías, que apostaba por una explicación pausada de la realidad que se enriquecía con hipervínculos de la más variada naturaleza. Dicha estructura principal se completaba con la opción de consulta a un gran banco de datos con alrededor de 60.000 ficheros que proporcionaban detalles a través de tres categorías de búsqueda: por países, personas que aparecían en los archivos o categoría de relato. El acceso a cualquiera de esos contenidos ofrecía al lector elementos adicionales en forma de mapas, gráficos de barras y texto descriptivo.

Bien distinta era la narrativa en el caso de Skin and Bone. Además de los aspectos propios de Swiss Leaks se optó por otorgar particular relevancia en el relato a dos piezas que resultaron particularmente impactantes (tanto para los asociados como para los consumidores finales): un vídeo de algo menos de diez minutos, en el que se describía de manera pormenorizada el proceso examinado (hasta tal punto que en su carátula de presentación se advertía acerca de la crudeza de las imágenes que estaban a punto de verse); y distintas fotografías del cuerpo humano donde se indicaban los diferentes tipos de operaciones quirúrgicas que se realizaban a partir del referido tráfico de tejidos humanos. 
Un caso aparte es el conocido como Papeles de Panamá, esbozado en el epígrafe anterior. Las dimensiones de lo que ha sido la mayor filtración periodística de la historia (alrededor de 11,5 millones de documentos que ocupaban 2,6 terabytes de información digital fechada entre 1977 y 2015) requerían una propuesta narrativa sustancialmente diferente a lo habitual en cuanto a la gestión de los datos se refiere.

Tras la limpieza y organización de la información, se pusieron dos plataformas al servicio del más de centenar de medios asociados: una, para consultar la documentación mediante buscadores llamada Blacklight, y otra, Linkurious, que proyectaba gráficos de relaciones y permitía comprender con mayor facilidad los complejos vínculos entre las redes offshore y sus beneficiarios. Dichos contenidos se compartían a través de la plataforma Global I-Hub, cuyo funcionamiento operativo era similar al de una intrared social. Aunque estas interfaces no se trasladaron a la comunidad de receptores, sí que permitieron que la iniciativa se mantuviera en secreto durante más de un año pese a implicar a gran cantidad de profesionales de la información.

El éxito obtenido por estos proyectos ha permitido incrementar los niveles de popularidad del International Consortium of Investigative Journalists y ha redundado de forma positiva en la obtención de fondos adicionales recibidos para reforzar sus planteamientos estratégicos de colaboración en periodismo de investigación bajo una faceta no lucrativa, así como para continuar utilizando formatos narrativos no convencionales.

Por indicar algunos aspectos meramente cuantitativos que permiten comprobar el grado de acogida de esta política estratégica, en los últimos tres años el ICIJ ha realizado una apuesta particularmente intensa para acceder a nuevas audiencias empleando el poder de las redes sociales. Según los datos correspondientes a finales de febrero de 2017 (ICIJ, 2017), el consorcio cuenta con más de 100.000 seguidores en Twitter y por encima de los 105.000 en Facebook y posee más de 14.500 suscriptores en su canal de YouTube. 


\subsection{ProPublica}

El empleo de nuevas narrativas resulta esencial dentro de la estructura productiva de ProPublica, entre otras cosas porque se convierte en uno de los pilares estratégicos para fomentar la viralidad de los contenidos y garantizar la viabilidad del proyecto.

Desde tal punto de vista la apuesta por relatos innovadores contribuye a facilitar que los donantes comprueben el impacto del dinero invertido (al aparecer buena parte de las informaciones generadas desde la redacción en grandes medios de comunicación) pero también hace posible que los socios publicadores se beneficien de investigaciones en profundidad (con un elevado valor de mercado, difíciles de conseguir en un contexto de reducción de redacciones y de incremento de costes económicos a todos los niveles) y que los socios difusores obtengan la ventaja competitiva de acceder a contenidos innovadores en su presentación sin necesidad de pagar por ello.

La generalización de reportajes multimedia en profundidad, en los que se combinan de manera sistemática textos, vídeos, fotografías e hipervínculos, ha fomentado un estilo gráfico que encuentra excelente acomodo, por ejemplo, en las audiencias locales, aportándolas contenidos de alcance nacional y transversal: "Cooperation with other medias turned out to breed an unexpected advantage: transforming good national stories into a local ones" (Filloux, 2012).

Un ejemplo significativo de esta propuesta estratégica puede ser el reportaje A Perfect Terrorist, David Coleman Headley's Web of Betrayal. En esta caso la colaboración se estableció con la cadena estadounidense de televisión PBS y aportó el valor añadido de cómo emplear recursos tecnológicos al alcance del usuario doméstico pero generando un relato periodístico de apenas seis minutos, narrado de manera innovadora, donde se mezclaban recursos de vídeos interactivos que eran ubicados mediante procedimientos clásicos impresos (pintándose sobre un soporte físico tipo pizarra transparente con un 
rotulador estándar) y que permitía su metas-eparación en pequeñas cápsulas de contenidos seleccionadas por la propia audiencia, junto a un grafismo sencillo y fácilmente comprensible.

El reportaje sentaba las bases para entender el papel jugado por David Coleman Headley en los atentados perpetrados en 2008 en Bombay en contra de Estados Unidos. Para su más clara comprensión, los principales items de su recorrido vital eran explicados con un breve texto puesto a disposición del receptor y en el que éste podía pinchar si así lo deseaba (y siempre en el orden en que lo decidiera, sin perderse en momento alguno el hilo narrativo). La pieza se completaba con un documental, de algo más de 53 minutos de duración, en este caso de carácter clásico.

Uno de los últimos retos que ha lanzado ProPublica se enmarca dentro de los delitos de odio. Su proyecto Colaboración para Seguir y Documentar Delitos de Odio a través de la inciativa Documentating Hate, lanzado a comienzos de marzo de 2017, pretende descubrir y publicar crímenes de odio con la ayuda de las redacciones de todo el país y va a desarrollar diferentes líneas de trabajo (Martin, 2017).

A todo ello se añaden, con particular intensidad a partir de mediados del año 2015 y cada vez con mayor peso específico, dos elementos adicionales: por un parte, los podcasts y, por otro lado, diferentes aplicaciones interactivas de la más variada naturaleza.

En el caso de los podcasts su utilización responde al de un soporte narrativo con entidad propia, no necesariamente incardinado en una estructura narrativa específica sino como elemento independiente y con valor informativo autónomo. Cada podcast dispone de un texto y de numerosos hipervínculos que permiten enriquecer su contenido y refuerzan dicha naturaleza autónoma. Por término medio, su duración oscila entre los quince y los veinte minutos, aunque no es infrecuente la aparición de casos excepcionales por su brevedad 
(incluso de dos tres minutos) o por su considerable extensión (por ejemplo una pieza generada en 2017 como The Breakthrough: What American Journalists Can Learn From Reporting Under Putin, supera la media hora de duración).

El segundo de los componentes que en los últimos meses viene siendo potenciado desde ProPublica es el empleo de aplicaciones interactivas (concebidas tanto para ordenadores de sobremesa como para dispositivos móviles) que permiten que el internauta construya, siempre de manera matizada y limitada por una estructura narrativa común, su propio relato.

Esta apuesta por las nuevas narrativas tiene su mirada también centrada en la optimización de su posterior difusión ya no sólo desde las páginas web de los cibermedios que difunden los contenidos, sino desde sus respectivas redes sociales. A finales de febrero de 2017 (ProPublica, 2017) la página web de ProPublica registraba un tráfico mensual de casi tres millones de páginas vistas con alrededor de un millón de visitantes únicos. Su número de seguidores era de más de 265.000 en Facebook y por encima de los 500.000 en Twitter. Además, sus suscriptores se situaban en 75.000 y sus donantes a título individual excedían los 700. También contaba con más de 6.700 suscriptores de su canal de YouTube.

\subsection{J ournalism++}

A la hora de considerar la apuesta por las nuevas narrativas realizada por Journalism++ es preciso tener en cuenta que, de los tres proyectos transnacionales aquí considerados, es el que ha ido más adelante en cuanto a la colaboración periodística se refiere. La iniciativa no se limita a compartir los contenidos generados sino que aboga por la producción colaborativa sustentada en diversos grupos de trabajo bajo pautas de trabajo consolidadas y con objetivos comunes. 
Dicha realidad implica unos procedimientos narrativos que, en apariencia, resultan menos innovadores como consecuencia de la necesidad de que cada uno de sus socios productores (en este caso quien asume funciones como la búsqueda de datos, el análisis de informaciones, la canalización de entrevistas con las fuentes y, en definitiva, la generación de material propio en función de los propósitos comunes previamente determinados) esté siempre bajo la supervisión y la tecnología de la organización.

Es desde la organización central desde la que se facilita el acceso a herramientas tecnológicas de máximo nivel y sofisticación, a las que difícilmente podría accederse desde otros medios de comunicación, aunque también es desde dicha organización donde se establecen los límites narrativos para generar los distintos relatos informativos.

Por la propia idiosincrasia de la iniciativa, Journalism ++ ha ido ampliando y perfeccionando la gama de herramientas de tecnologías de la información y comunicaciones que pone al servicio del periodismo de investigación en general y de sus socios productores en particular. A efectos prácticos está consolidación TIC se percibe en una visualización de datos más eficaz.

Es cierto que el uso de herramientas de visualización de datos no es una práctica periodística tan novedosa como pudiera suponerse. Ya en 1952 la cadena televisiva estadounidense CBS utilizó un prototipo para pronosticar, con pleno acierto, los resultados de las elecciones presidenciales que otorgaron la victoria al candidato del Partido Repúblicano, Dwight Eisenhower. No obstante, la apuesta estratégica de Journalism ++ radica en un incremento cualitativo de los niveles de eficiencia de esta clase de innovación, lo que se concreta en el desarrollo e implantación de herramientas como Datawrapper, Hightcharts Cloud o Carto, entre otras.

Tal y como está aquí concebida, la visualización de datos simplifica la densidad de lectura inherente a contenidos compuestos por incluso miles de archivos 
(en programas Excel y similares), prácticamente incomprensibles para cualquier internauta de formación media. De manera adicional, la propuesta por nuevas narrativas de Journalism++ se ve reforzada por el sistemático empleo de software para la publicación de grandes bases de datos, como puede ser Detective.io o similares (por lo general en el ámbito de las plataformas de código abierto).

Quizá por lo hasta ahora indicado, a diferencia de las otras dos experiencias analizadas la apuesta estratégica por nuevos formatos narrativos no se corresponde con un uso particularmente intenso de las posibilidades del entorno Web 2.0. Con datos cerrados a febrero de 2017 (Journalism++, 2017), su número de seguidores en Facebook no llega a los 2.000 mientras que el de Twitter apenas alcanza los 5.600, cifras muy escasas en comparación con los respectivos proyectos de ICIJ y ProPublica.

\section{Discusión y conclusiones}

Las distintas iniciativas de periodismo de colaboración analizadas en este artículo surgen para dar respuesta a un entorno caracterizado por una pérdida de credibilidad de los medios de comunicación ante la opinión pública en general y los consumidores de sus contenidos en particular, así como a la creciente necesidad de financiación para acometer proyectos que requieren una considerable cantidad de recursos económicos y humanos. Este entorno se ve particularmente influido por la convergencia de factores como la consolidación de un nuevo estándar en la adquisición/transmisión del conocimiento, el paso de un mercado de demanda a otra de oferta en el negocio de la información, el afianzamiento de las NTIC en la producción, almacenamiento y distribución de contenidos y el desplazamiento del consumidor de información en beneficio de la figura del prosumidor, a la que ya se ha aludido al inicio del texto, entre otros.

De manera adicional, el paso hacia la emergente Web 2.0 convierte Internet en una mega ágora, una gran virtual town square (Gillmor, 2005: 11) donde las 
audiencias de todo el mundo establecen una conversación a escala global en la que comparten conocimientos, inquietudes, experiencias y contenidos y donde las empresas de la información transforman sus procedimientos de actuación y la presentación de sus contenidos a dichas audiencias. Este emergente contexto propicia las iniciativas colaborativas en el ámbito de la información, con particular relevancia en lo que atañe al sub-segmento del periodismo de investigación, aportando ventajas competitivas como la optimización de sus considerables costes económicos o un incremento en sus niveles de impacto social sobre una opinión pública cada vez más globalizada.

A fecha de hoy, propuestas específicas de actuación como las de International Consortium of Investigative Journalists, ProPublica o Journalism ++ prueban la creciente solidez del periodismo colaborativo de investigación, así como la diversidad de tipologías que admite dicho formato: desde la mera compartición de contenidos hasta la apuesta por el desarrollo de acciones integradas colectivas, pasando por la sistematización en cuanto a la producción de narraciones periodísticas se refiere; desde la asunción por parte de organizaciones nativas digitales (como son los casos de ProPublica y Journalism ++ ) hasta su implantación en organizaciones surgidas incluso con anterioridad a la eclosión de Internet (conforme a lo que ocurre con International Consortium of Investigative Journalists); desde la puesta en marcha de iniciativas sin ánimo de lucro (ICIJ y ProPublica) hasta la propuesta de organizaciones lucrativas (Journalism++); desde el empleo activo de las redes sociales como herramienta estratégica de difusión de los contenidos (International Consortium of Investigative Journalists y ProPublica) hasta el empleo meramente testimonial de las mismas (Journalism++).

La apuesta por las nuevas narrativas que desarrollan toda esta clase de proyectos periodísticos se sustenta en dos ejes estratégicos fundamentales: por una parte, una propuesta multimedia en lo formal con un diferente estilo narrativo en cuanto al contenido se refiere; y, por otro lado, un conjunto de nuevos procedimientos relativos a los procesos de obtención, gestión, 
tratamiento y presentación formal de las ingentes cantidades de datos que son necesarias para generar contenidos periodísticos de calidad en forma de reportajes de investigación en profundidad. Ambas facetas tienen como nexo común la activa aplicación de las nuevas tecnologías de la información y las comunicaciones, concretada en el empleo tanto de herramientas de software de carácter propietario como de otras sustentadas en código de acceso libre.

\section{Bibliografía}

Berry, A. (2016). ICIJ to Separate from Center for Public Integrity.

Recuperado de https:// nonprofitquarterly.org/2016/10/24/icij-separatecenter-public-integrity/.

Bird, S. E. (2011). Are We All Producers Now? Convergence and Media Audience Practices. Cultural Studies, 25, (4-5), 502-516. Recuperado de http://www.tandfonline.com/doi/full/10.1080/09502386.2011.600532.

Bradshaw, P. (2008). Investigative Journalism and blogs. En H. De Burgh (Ed.), Investigative Journalism, pp. 96-113. Nueva York: Routledge.

Bruns, A. (2009). From Prosumer to Produser: Understanding User-Led Content Creation. En Transforming Audiences, Londres, pp. 1-18. Recuperado de https://www.slideshare.net/Snurb/from-prosumer-toproduser-understanding-userled-content-creation.

- (2006). Towards Produsage: Futures for User-Led Content Production. En F. Sudweeks, H. Hrachovec y C. Ess (Eds.), Cultural attitudes towards technology and communication 2006 : proceedings of the Fifth International Conference on Cultural Attitudes Towards Technology and Communication, pp. 275-284, Tartu: Murdoch, W.A. 
Cluetrain. (1999). The Cluetrain Manifesto. Recuperado de http://www.cluetrain.com.

Díaz Nosty, B. (2013). La prensa en el nuevo ecosistema informativo. ¡Que paren las rotativas! ( $1^{\mathrm{a}}$ ed.) Barcelona: Ariel/Fundación Telefónica.

Dinucci, D. (1999). Fragmented Future. Print Magazine, 53, (4), 32, 221-222. Recuperado de http://darcyd.com/fragmented_future.pdf.

Filloux, F. (2012). Lessons from ProPublica. Monday Note. Recuperado de http://www.mondaynote.com/2012/07/01/lessons-from-propublica.

García Galera, M.C. y Del Hoyo, M. (2013). Redes sociales, un medio para la movilización juvenil. Zer Revista de Estudios de Comunicación, 34, (18), 111 125. Recuperado de http://www.ehu.eus/zer/hemeroteca/pdfs/zer34-06garcia.pdf.

Gershenfeld, N., Krikorian, R. y Cohen, D. (2004). The Internet of Things. Scientific American, 291, (4), 76-81. Recuperado de http://cba.mit.edu/docs/papers/04.10.i0.pdf.

Gómez Mompart, J.L., Gutiérrez Lozano, J.F. y Palau, D. (2015). Los periodistas españoles y la pérdida de calidad de la información: el juicio profesional. Comunicar, 45, (22), 143-150. DOI: http://dx.doi.org/10.3916/C45-2015-15.

Hunter, M.L. (2013). La investigación a partir de historias. Manual de periodismo de investigación. (1 ${ }^{\mathrm{a}}$ ed.) Montevideo: Unesco. ICIJ. (2017). International Consortium of Investigative Journalists. Recuperado de http://www.icij.org. 
Irala, P. (2014). Nuevas narrativas en el periodismo actual. El periodismo transmediático. Estudios sobre el Mensaje Periodístico, 20, (1), 147-158. DOI: http://dx.doi.org/10.5209/rev_ESMP.2014.v20.n1.45224.

Jenkins, H. (2009). The Revenge of the Origami Unicorn: Seven Principles of Transmedia Storytelling. Recuperado de:

http://henryjenkins.org/2009/12/the_revenge_of_the_origami_uni.html.

Journalism++. (2017). Journalism++ Manifesto. Recuperado de http://www.jplusplus.org/en/manifesto/.

Martin, M. (2017). ProPublica Launches New Collaboration Project To Track And Document Hate Crimes. Recuperado de http:/ /www.npr.org/2017/03/05/518663829/propublica-launches-newcollaboration-project-to-track-and-document-hate-crimes.

Moloney, K. (2011). Transmedia Journalism in Principle. Recuperado de: https://transmediajournalism.org/2011/11/23/transmedia-journalism-inprinciple/.

O'Reilly, T. (2005). What is Web 2.0. Design Patterns and Business Models for the Next Generation of Software. Recuperado de http://www.oreilly.com/pub/a/web2/archive/what-is-web-20.html.

Parasie, S. (2015). Data-Driven Revelation? Epistemological tensions in investigative journalism in the age of 'big data'. Digital Journalism, 3, (3), 364380. Recuperado de http://www.tandfonline.com/doi/full/10.1080/21670811.2014.976408.

ProPublica. (2017). ProPublica Financial Statements December 31, 2016. Recuperado de http://propublica.s3.amazonaws.com/assets/about/2015- 
Pro-Publica-Inc-

Financial\%20Statements.pdf?_ga =1.36931296.1567246357.1475061433.

Ryle, G. (2017). After Panama Papers success, ICIJ goes independent.

Recuperado de: https://www.icij.org/blog/2017/02/after-panama-paperssuccess-icij-goes-independent.

Sabés, F. y Parra, D. (2014). Del consumidor al prosumidor: responsabilidades legales de los informers. El profesional de la información, 23, (5), 511-518. DOI: http://dx.doi.org/10.3145/epi.2014.sep.08.

Scolari, C.A. (2013). Narrativas transmedia. (1ª ed.) Barcelona: Deusto.

Shirky, C. (2008). Here Comes Everybody: The Power of Organizing without Organizations. ( $1^{\mathrm{a}}$ ed.) Nueva York: Penguin Group.

Toffler, A. (1990). Powershift Knowledge, Wealth and Violence at the Edge of the 21 st Century. Nueva York: Bantam.

World Association of Newspapers and News Publishers. (2015). World Press Trends 2015. Recuperado de http://www.wan-ifra.org/pressreleases/2015/06/01/world-press-trends-newspaper-revenues-shift-to-newsources.

\section{Financiación}

Este artículo forma parte de los trabajos llevados a cabo en el marco del proyecto de investigación "Claves para la redefinición y supervivencia del periodismo y retos en la era post-PC. Medios emergentes, nuevas narrativas, agregadores, robots, multiscreen, first mobile, apps” (Ref. CSO201679782-R). Dicho proyecto cuenta con la financiación del Ministerio de Economía, Industria, y Competitividad (MINECO) del Gobierno de España a través del Plan Nacional de I+D+i dentro del Programa Estatal de Investigación, Desarrollo e Innovación Orientada a los Retos de la Sociedad. 


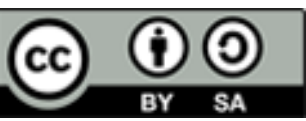

Licencia Creative Commons

Miguel Hernández Communication Journal

mhjournal.org

\section{Cómo citar este texto:}

David Parra Valcarce, Concha Edo Bolós y Juan Carlos Marcos Recio (2017): "Nuevas narrativas aplicadas al periodismo colaborativo de investigación”, en Miguel Hernández Communication Journal, n8, pp. 591 a 616. Universidad Miguel Hernández, UMH (ElcheAlicante). Recuperado el $\_$de mhjournal.org] de 20_ de: link del artículo en 